\title{
Fourth annual North American and 1990 international cystic fibrosis conference
}

\author{
J S Elborn
}

The discovery of the cystic fibrosis gene over a year ago has resulted in intensive research to identify all the mutations that cause this disease, to investigate the potential of gene therapy, and to determine the exact nature and function of the normal gene product. Many of the advances since the discovery of the gene were described at the Fourth North American and 1990 International Cystic Fibrosis Conference in Washington DC in October to an audience of over 2000, including 20 clinicians and scientists from Eastern Europe. The emphasis of the meeting was on developments in molecular and cell biology in relation to cystic fibrosis, but there was also much to interest clinical and paramedical staff concerned with the care of patients with cystic fibrosis. The proceedings of the meeting are published as supplement 5 (1990) in Pediatric Pulmonology.

\section{Molecular and clinical genetics}

In September 1989 Tsui, Collins, and Riordan described the $4 \mathrm{~F} 508$ mutation of the cystic fibrosis gene. Since then a "gene consortium" of over 20 laboratories under the direction of Dr Tsui have shared information and gene probes. These groups have described over 60 mutations of the gene, which include other base triplet deletions and mis-sense and nonsense changes that often result in truncation of the transmembrane regulator protein that is defective in cystic fibrosis (known as the cystic fibrosis transmembrane regulator protein). Most mutations affect the nucleotide binding zones of the cystic fibrosis transmembrane regulator, which bind ATP, suggesting that the gene product takes part in an active transport mechanism.

Studies presented from various centres showed only a weak link between the genotype and the phenotype in cystic fibrosis. Several groups reported that the most common mutation in white patients, $\Delta \mathrm{F} 508(75-80 \%)$, is not predictive of the severity of lung disease. Almost all patients homozygous for $\triangle \mathrm{F} 508$ have pancreatic insufficiency, whereas those who have pancreatic function preserved either are heterozygous for $\Delta \mathrm{F} 508$ or carry less common mutations. It was also suggested that $\triangle F 508$ homozygotes have higher resting energy expenditure than heterogyzotes and those with other mutations, raising the possibility that the $\triangle \mathrm{F} 508$ mutation may be associated with less efficient use of energy at a cellular level.
The implications of the already large and increasing number of known mutations of the cystic fibrosis gene for family and population screening were discussed. There is considerable racial variation in the occurrence of mutations. For example, the proportion of cases caused by $\triangle \mathrm{F} 508$ was reported to increase from $40 \%$ to $85 \%$ between south east and north west Europe. In North America this mutation is common in some ethnic groups, such as the Amish and Hutterites, but relatively uncommon in Ashkenazi Jews. These racial variations and the large number of rare mutations raise the question of how many mutations should be screened for in a particular population. Screening for a few would result in a relative rather than an absolute risk for a given pregnancy, and it was proposed that for any population a 90-95\% predictive ability is necessary to justify a population based screening programme. This may be possible in Britain by screening for three to five mutations, as $\Delta \mathrm{F} 508$ is present in $75-80 \%$ of patients with cystic fibrosis. Some pilot experience of population screening was reported from a study based on general practices in southern England. Eighty five per cent of adults of reproductive age offered screening through their family doctor or family planning clinic had little or no knowledge of the nature of cystic fibrosis. The best uptake of screening occurred in patients who were opportunistically offered the screening test when attending their general practitioner, local baby clinic, or family planning clinic for other reasons. Disappointingly, less than $20 \%$ responded to a letter inviting them to attend for a screening test. Less than $40 \%$ of all the women screened said that they would definitely have a termination of their pregnancy if found to be carrying an affected fetus. These results raise the question of whether population screening will be logistically feasible, cost effective, and acceptable to the general public. The effect of litigation, particularly in the United States, may be a further complication.

Structure and function of the cystic fibrosis transmembrane regulator

The structure and function of the gene product, the cystic fibrosis transmembrane regulator protein, is still not elucidated. Chloride ion transport in cystic fibrosis epithelium has been known for some years to be abnormal and when the protein was sequenced it seemed possible that it might be an active chloride transporter. The evidence,
Correspondence to: Respiratory Medicine Unit, City Hospital, Nottingham NG5 $1 \mathrm{~PB}$. 
however, shows fairly conclusively that the cystic fibrosis transmembrane regulator is not a chloride channel. In people without cystic fibrosis it is present at low levels even in epithelial cell membranes such as respiratory mucosa, and mRNA is expressed at a level consistent with its being a housekeeping gene-that is, one copy a cell. The cystic fibrosis transmembrane regulator protein belongs to a "superfamily" of membrane transporters, all of which are made up of nucleotide binding folds and transmembrane domains. It has two nucleotide binding folds that bind ATP and six transmembrane domains, and is similar to the $\mathbf{P}$ glycoprotein multiple drug transporter responsible for resistance in cultured cells to hydrophobic chemotherapeutic agents. Probably therefore the cystic fibrosis transmembrane regulator actively transports a lipid derived mediator such as an eicosanoid or cytokine and this is coupled in some manner to regulate chloride transport. It was clear that much work is still required to elucidate the function of the cystic fibrosis transmembrane regulator and how it relates to ion transport. A protein with $80 \%$ homology with the cystic fibrosis transmembrane regulator has been isolated from the shark rectal gland, which is an important organ in salt homeostasis. It is hoped that this and other conserved homologous proteins in other species may shed further light on the function of the cystic fibrosis transmembrane regulator.

\section{Gene therapy}

Perhaps the most exiting development since the description of the gene was reported in work from several laboratories in the United States that are exploring in vitro gene therapy. Professor RJ Crystal from the National Institute of Health, Bethesda, described ex vivo respiratory cell lines and in vivo animal models into which exogenous genes, such as the $\alpha_{1}$ antitrypsin gene, have now been transferred. His group and others described gene transfer to cystic fibrosis cell lines with a retroviral vector. The introduction of DNA in this manner at a level of one to two copies a cell was shown by isotopic anion (iodine-125) efflux to correct the chloride channel defect, thought to be the underlying physiological abnormality. This is an exciting development and moves the possibility of gene therapy a step forward. It also confirms that the cystic fibrosis transmembrane regulator protein regulates chloride ion transport, though the mechanism still requires elucidation. Major questions remain regarding the delivery of retrovirus or other vectors, stability of DNA within vectors, expression of DNA in vivo, and potential cellular toxicity. A genetic cure for cystic fibrosis is probably still many years away.

\section{Lung disease}

The sessions devoted to lung disease focused on mechanisms of injury, particularly in the context of Pseudomonas aeruginosa infection. The importance of neutrophil recruitment and the subsequent imbalance of the elastaseantielastase ratio in the lung were considered.
The possible use of antiproteases, such as $\alpha_{1}$ antiproteinase, or a secretory leucoprotease inhibitor to reduce elastase activity were discussed. In an open study of 12 patients with cystic fibrosis nebulised recombinant $\alpha_{1}$ antiproteinase treatment for seven days resulted in a reduction in the purulence and volume of sputum and an improvement in spirometric values and gas transfer. Further studies will be necessary but these compounds are likely to be helpful in managing lung disease in cystic fibrosis. Other systemic anti-inflammatory treatments were suggested. The role of steroids and non-steroidal anti-inflammatory agents are currently under investigation but no firm evidence was presented to support their use. One small though controlled study suggested that hospital admissions with respiratory exacerbation were reduced with regular treatment with the non-steroidal anti-inflammatory drug piroxicam.

The role of inflammatory cytokines such as tumour necrosis factor alpha and interleukins 1 and 6 was also discussed and some evidence was advanced to suggest that they might be important in mediating pulmonary inflammation in cystic fibrosis. These cytokines may perpetuate the chronic inflammatory response, causing further lung damage, and they may contribute to the progressive weight loss seen in the later stages of disease. Regulation of the cytokine network was suggested as a potentially useful approach that might attenuate local inflammatory processes and reduce lung damage.

\section{Nutrition and energy}

Few new developments in nutritional support were reported. The importance of accurate assessment of nutritional state was emphasised, though most centres will not be able to undertake detailed measurements of metabolic rate as suggested. Although resting energy expenditure is raised in cystic fibrosis, total daily energy expenditure was reported to be similar to that of control subjects. Patients with cystic fibrosis have a reduced level of daily activity to compensate for their increased resting metabolic rate. Diabetes and hepatic complications were also discussed but no important developments in these areas were reported.

Many nursing, paramedical, psychological, and social issues were discussed and these sessions were particularly helpful for the many non-clinical care givers attending the conference. An increasing enthusiasm was evident among nurses and others for critically evaluating their practice. This first major conference after the discovery of the gene was stimulating and challenging. Many questions on the function of the cystic fibrosis transmembrane regulator, the application of gene therapy, and the role, if any, of population screening have yet to be answered. A gene therapy cure for the membrane defect is at least five and maybe 10 years away, but it is encouraging and exciting to know that the membrane defect can be corrected in vitro. In the meantime there is much to be done in caring for those patients who have already substantial lung disease and ways to minimise lung damage must be actively pursued. 